\title{
Puerulus y postpuerulus de Projasus bahamondei George, 1976 (Crustacea, Decapoda, Palinuridae)
}

\author{
Pedro Báez R. y Rosario Ruiz L. \\ Sección Hidrobiología, Museo Nacional de Historia Natural \\ Interior Parque Quinta Normal. Casilla 787, Santiago \\ E-mails: pbaez@mnhn.cl y rruiz@mnhn.cl
}

Recibido: 12 abril 1999; versión corregida: 9 agosto 1999; aceptado: 21 noviembre 1999.

\begin{abstract}
RESUMEN: Se describen estadios de puerulus y postpuerulus de la langosta espinosa de profundidad, n.v.: langosta de Valparaíso, Projasus bahamondei. El material fue obtenido en pescas de arrastre comerciales de langostinos y camarón nailon. Existe variabilidad entre los distintos ejemplares de larvas y postlarvas estudiados. El tamaño de los ejemplares es mayor que el puerulus de otras especies de palinúridos. P. bahamondei, del talud continental de Chile, archipiélago de Juan Fernández, islas Desventuradas y del cordón submarino de Nazca, junto a P. parkeri de Sudáfrica, Nueva Zelanda e isla Saint Paul en el océano Indico y Projasus sp. de New South Wales, Australia, pertenecen a un género circumpolar de profundidad, filogenéticamente muy antiguo, de origen Gondwánico. Se comparan los estadios descritos con aquéllos de P. parkeri y Projasus sp.
\end{abstract}

Palabras claves: Palinuridae, Projasus bahamondei, langosta espinosa, puerulus, postpuerulus, Pacífico suroriental.

\section{Puerulus and postpuerulus of Projasus bahamondei George, 1976 (Crustacea, Decapoda, Palinuridae)}

\begin{abstract}
The puerulus and the postpuerulus stages of Projasus bahamondei, common name: Langosta de Valparaíso are described. The material was collected from the deep fisheries off Central Chile by trawlings in the captures of squat lobsters and shrimps. A certain variability was observed among the studied larval specimens and the postlarval ones. The size of these specimens are in general larger than the pueruli and postpueruli described form other palinurid species. P. bahamondei from the Chilean continental slope, Juan Fernandez archipelago and Desventuradas islands, and from the Nazca Ridge, together with P. parkeri from South Africa, New Zealand and Saint Paul Island from the Indian Ocean, and Projasus sp. from New South Wales, Australia, belong to a circumpolar genus, very old phylogenetically, which has a Gondwanic origin. The described stages of $P$. bahamondei are compared with those from P. parkeri and Projasus sp.
\end{abstract}

Key words: Palinuridae, Projasus bahamondei, spiny lobsters, puerulus, postpuerulus, Southeastern Pacific.

\section{INTRODUCCIÓN}

La familia Palinuridae está representada en aguas chilenas por tres especies. Projasus bahamondei descrita por George (1976), se distribuye en el archipiélago de Juan Fernández (Retamal y Arana, 2000), islas San Félix y San Ambrosio, así como también en profundidad frente a Iquique y en la zona arquibéntica frente a la costa de Chile continental, entre Huasco y Constitución (175-550 m). También ha sido reportada en el cordón submarino de Naz- ca, a unas 400 millas de la costa sudamericana, frente al límite entre Chile y Perú (Weinborn et al., 1992; Retamal y Arana, 2000). Jasus frontalis (H. Milne Edwards, 1837), vive en el archipiélago de Juan Fernández e islas San Félix y San Ambrosio, y Panulirus pascuensis Reed, 1954, habita en las islas de Pascua y Pitcairn (Polinesia inglesa).

La información existente referente a los estadios larvarios y postlarvarios de las tres especies es aún 
muy reducida. De P. bahamondei existía sólo la descripción de una larva phyllosoma, asignada primero y tentativamente a $P$. parkeri por Báez (1973). Se sigue desconociendo aún el resto de las larvas phyllosoma y las transformaciones de su metamorfosis. Especialmente importantes son los cambios finales que le ocurren a estas larvas, los cuales son la expresión de los cambios de hábitat y de comportamiento desde una vida completamente planctónico-pelágica a la béntica, característica de los adultos.

\section{MATERIAL EXAMINADO}

Se examinaron seis especímenes de Projasus bahamondei (Tabla 1): dos puerulus y cuatro postpuerulus. Estos ejemplares fueron recolectados en pescas comerciales del camarón nailon (Heterocarpus reedi) y de los langostinos colorado (Pleuroncodes monodon) y amarillo (Cervimunida johni), obtenidos con artes de arrastre frente a la zona central de Chile. Junto a numerosos invertebrados esta especie constituía parte de la fauna acompañante de estas capturas. El material, fijado en formalina $(7 \%)$ y preservado en alcohol $\left(70^{\circ}\right)$ fue incorporado a la colección de crustáceos del Museo Nacional de Historia Natural de Santiago (MNHNCD). Morfometría: Longitud total; longitud del caparazón; ancho del caparazón; longitud del pleon; longitud de la anténula; longitud del pedúnculo de la antena; y, patas ambulatorias 1 a 5, medidas sólo las derechas. La longitud total y cefalotorácica se midieron desde la punta del rostro al margen posterior en la línea media del telson y del caparazón, respectivamente. El ancho del caparazón se midió a

Tabla 1. Projasus bahamondei. Datos de recolección y morfometría ( $\mathrm{mm})$.

Table 1. Projasus bahamondei. Collection data and morphometry (mm).

\begin{tabular}{|c|c|c|c|c|c|c|}
\hline $\begin{array}{c}\text { Ejemplar } \mathbf{N}^{\circ} \\
(\text { MNHNC D) } \\
\text { Datos y medidas }\end{array}$ & 11097 & $\begin{array}{c}11098 \\
\text { (Fig. 1a) }\end{array}$ & $\begin{array}{c}11099 \\
\text { (Fig. 2a) }\end{array}$ & 11106-a & 11106-b & 11106-c \\
\hline Localidad & Zapallar & Zapallar & Zapallar & El Teniente & El Teniente & El Teniente \\
\hline Latitud $S$ & $32^{\circ} 32^{\prime}$ & $32^{\circ} 32^{\prime}$ & $32^{\circ} 32^{\prime}$ & $30^{\circ} 59^{\prime}$ & $30^{\circ} 59^{\prime}$ & $30^{\circ} 59^{\prime}$ \\
\hline Longitud W & $71^{\circ} 40^{\prime}$ & $71^{\circ} 40^{\prime}$ & $71^{\circ} 40^{\prime}$ & $71^{\circ} 55^{\prime}$ & $71^{\circ} 55^{\prime}$ & $71^{\circ} 55^{\prime}$ \\
\hline Profundidad (m) & 350 & 300 & 350 & 400 & 400 & 400 \\
\hline Fecha & 27 Sep. 77 & 18 Nov. 76 & 27 Sep.77 & 16 Jun. 78 & 16 Jun. 78 & 16 Jun. 78 \\
\hline Recolector & A. González & A. González & A. González & S. Zunino & S. Zunino & S. Zunino \\
\hline Estadio desarrollo & Puerulus & Puerulus & Postpuerulus & Postpuerulus & Postpuerulus & Postpuerulus \\
\hline Longitud total & 50,3 & 48,9 & 58,4 & 52,9 & 59,3 & 57,7 \\
\hline Longitud caparazón & 20,3 & 19,9 & 22,5 & 21,8 & 23,9 & 23,6 \\
\hline Ancho caparazón & 11,4 & 11,6 & 13,3 & 12,7 & 14,6 & 13,8 \\
\hline Longitud anténula & - & - & - & 21,7 & 23,7 & 22,9 \\
\hline Longitud pedúnculo antena & 16,8 & 15,9 & 20,3 & 16,7 & 20,1 & 19,4 \\
\hline Longitud del pleón & 29,5 & 29,9 & 35,9 & 31,1 & 35,4 & 34,1 \\
\hline Pata ambulatoria 1 & $*$ & 23,6 & 23,9 & 21,0 & 25,1 & 24,7 \\
\hline Pata ambulatoria 2 & $*$ & - & 30,3 & - & 29,9 & 30,0 \\
\hline Pata ambulatoria 3 & $*$ & 27,8 & 31,2 & - & 32,9 & 32,3 \\
\hline Pata ambulatoria 4 & $*$ & 27,5 & 32,7 & - & 33,8 & 31,0 \\
\hline Pata ambulatoria 5 & $*$ & 26,5 & -- & 29,0 & 33,1 & -- \\
\hline
\end{tabular}

*= patas incompletas y dañadas 
través de la región torácica en su punto más amplio. $P$. bahamondei se incluye en las categorías taxonómicas:

Clase Malacostraca Latreille, 1806

Subclase Eumalacostraca Grobben, 1892

Orden Decapoda Latreille, 1803

Infraorden Palinura Latreille, 1803

Familia Palinuridae Latreille, 1803

\section{Descripciones}

Estadio puerulus (Fig.1a). MNHNC D - N 11098. Ejemplar con cutícula traslúcida, en algunos sectores transparente; de color café anaranjado, café claro en el abdomen y tonos chocolate en el caparazón. De forma similar a las langostas adultas. Espinas y setas relativamente romas y frágiles, no totalmente proyectadas fuera de la superficie corporal.

Caparazón subrectangular, con un rostro corto y robusto de posición central y anterior. Superficie dorsal relativamente aplanada, lisa y suave en su mayor extensión. Una depresión transversal delimita las regiones gástrica y cardíaca; otra en el tercio medio posterior enmarca la elevación del borde posterior; a ambos lados una depresión longitudinal, ligeramente encorvada delimita la región cardíaca central de cada región pleural lateral.

Región anterior del caparazón con un par de espinas o cuernos supraoculares robustos dirigidos hacia arriba y adelante, con márgenes anteriores casi rectos, suavemente encorvados, coronados por una espina aguzada. Existe un segundo par de espinas, mucho más pequeñas, ubicado inmediatamente detrás de los cuernos y en línea recta con éstos; otro par de espinas similares a estas últimas se ubica lateralmente detrás de las órbitas y otro par más pequeño es de posición más central. Estas últimas forman a cada lado, con los cuernos supraorbitales y las espinas que se ubican inmediatamente detrás de aquéllos, una línea ligeramente oblicua. Los primeros dos pares de espinas situadas en las filas submedianas sobre la región gástrica, están separados de las anteriormente señaladas por una espina central pequeña; a la vez se encuentran a una cierta distancia de los seis pares de espinas paralelas siguientes. Existen dos espinas anterolaterales robustas y dirigidas hacia afuera y adelante; éstas quedan separadas de las grandes espinas dorsolaterales únicas situadas a cada lado debido a la existencia de una constricción del caparazón, a ese nivel. Detrás de las dorsolaterales se dispone una fila longitudinal, ligeramente encorvada, de 8-9 espinas más pequeñas, las que con una orientación longitudinal se extienden sobre las regiones pleurales. Elevación transversal posterior del caparazón con una fila de tubérculos muy pequeños.

Esternito cefalotorácico subtriangular, provisto de una espina robusta frente a la base de cada una de las patas ambulatorias; estas espinas son más grandes hacia las patas posteriores. Existen cuatro dientes o espinas que se ubican en la línea media central del cefalotórax desde la primera a la cuarta pata ambulatoria. Borde posterior con dos espínulas centrales muy pequeñas.

Abdomen con una carina media dorsal. En los segmentos abdominales ésta queda demarcada por una espina roma de base ancha dirigida hacia arriba en el primero; una espina orientada hacia adelante en el segundo; cuarto, quinto y sexto segmentos con una espina media distal orientada hacia atrás. Superficie dorsal del sexto segmento provista de un par de espinas de orientación transversal; borde posterior con dos pares de espinas de ubicación posterolateral, de los cuales las más externas son más desarrolladas. Regiones pleurales ligeramente elevadas. El primer segmento abdominal es de bordes lisos, proyectado en un lóbulo hacia adelante; el segundo segmento presenta en el borde anterolateral un tubérculo robusto; dos dientes espinosos se dirigen hacia abajo y se encorvan hacia atrás. Los segmentos tercero al sexto mantienen el par de dientes laterales robustos y espinosos dirigidos hacia atrás, tornándose más aguzados en el penúltimo y último. Superficie de los tergitos lisa y suave, similar a aquélla del caparazón. Algunas setas plumosas se ubican en la parte posterior de los dientes pleurales del último y penúltimo segmentos.

Esternito del primer segmento abdominal con bordes rectos y un par de espinas sublaterales presentes; esta misma condición se presenta en el sexto segmento, aunque las espinas no son tan aguzadas como en el primero.

Telson con cuatro espinas pequeñas dispuestas en una línea oblicua, orientada desde el centro y hacia afuera; con bordes distales lisos. Se observan por transparencia numerosas líneas de tejido interno, dispuestas en un patrón de orientación longitudinal. Urópodos con exopodito provisto de 2-3 espinas en la base, orientadas hacia atrás. 

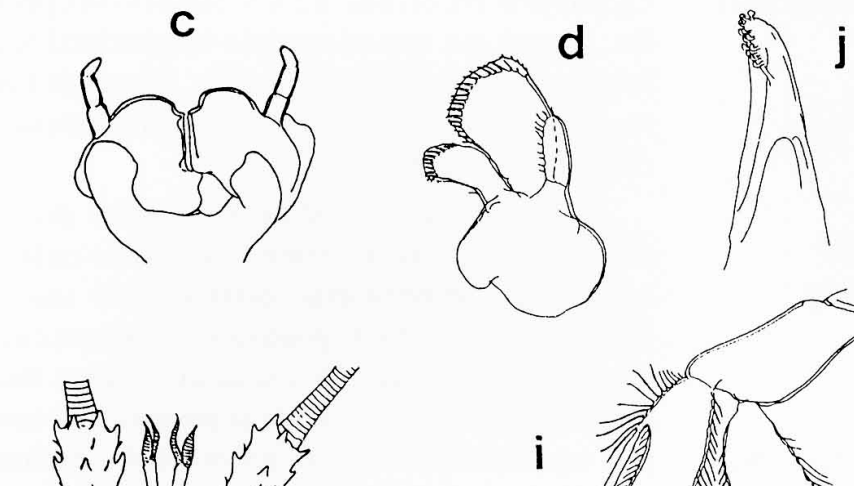

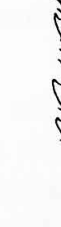
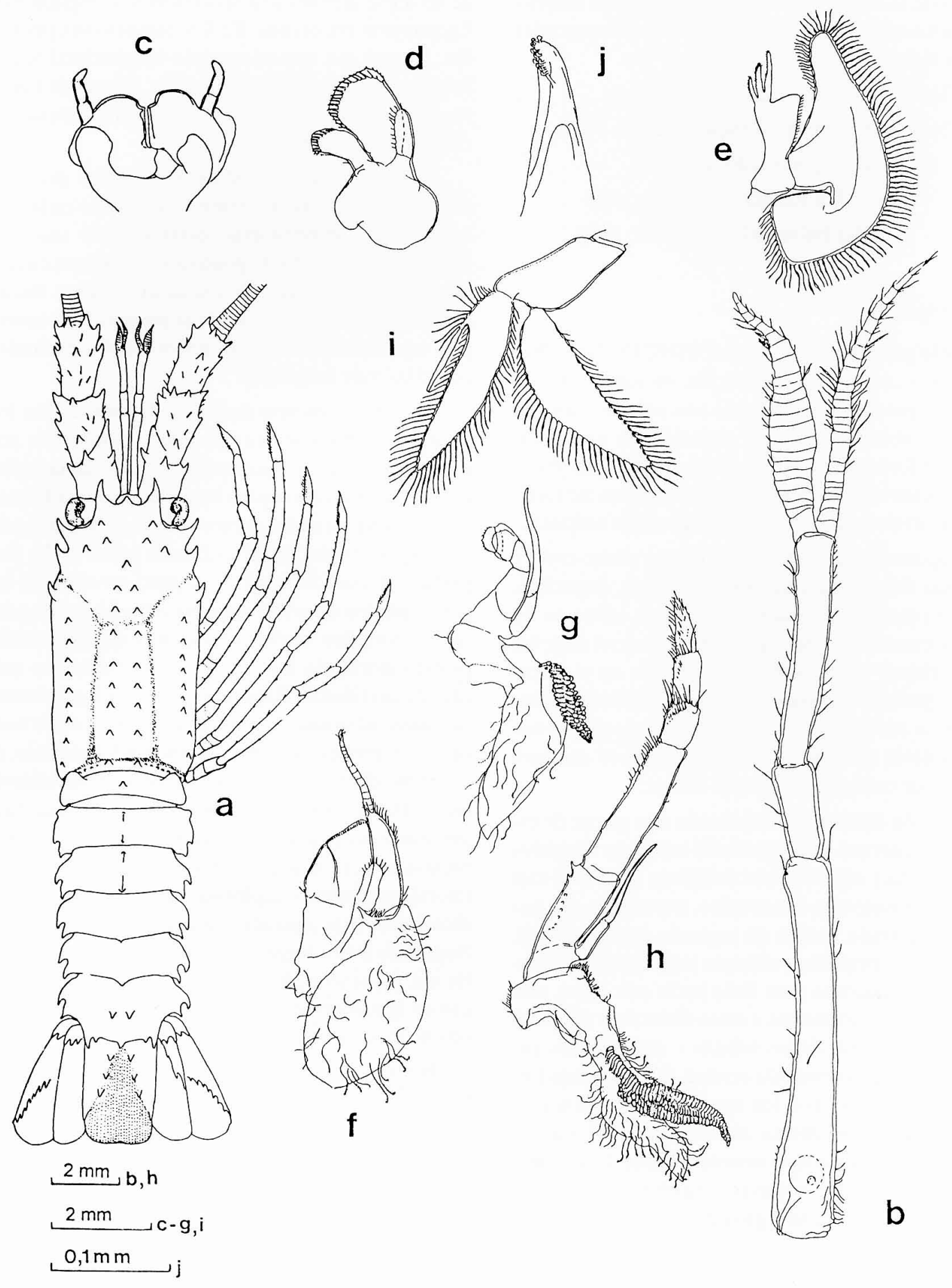

Figura 1. Projasus bahamondei: a, Puerulus en vista dorsal; b, anténula; c, mandíbula; d, maxílula; e, maxila; f, primer maxilípedo, g, segundo maxilípedo; h, tercer maxilípedo; i, pleópodo; j, apéndice interno del pleópodo.

Figure 1. Projasus bahamondei: a, Puerulus in dorsal view; b, antennule; c, mandible; d, maxillule; e, maxille; f, first maxilliped; g, second maxilliped, $h$, third maxilliped, $i$, pleopod; $j$, pleopod inner appendix. 
Cabe indicar que el pedúnculo ocular del puerulus es liso.

Anténulas (Fig.1b) cilíndricas y alargadas. El borde distal del segundo segmento coincide con aquél del segundo segmento de la antena. Los flagelos sobrepasan ligeramente el borde distal del tercer segmento antenal. Superficie del pedúnculo lisa, con escasas espinas y setas, todas muy espaciadas. El segmento proximal del pedúnculo está provisto de estatocisto basal; la longitud del segmento equivale a 3,5 veces el largo del segmento medio. El flagelo externo, que es más corto y ancho, presenta 22-23 segmentos, los que desde el sexto están provistos de setas en la región distal lateral; el interno en cambio posee 25-26 segmentos de los cuales a partir del séptimo segmento están provistos de setas en los bordes laterodistales.

Antenas (Fig.1a). Mucho más largas que el cuerpo, con flagelo cilíndrico que termina en punta aguzada. Pedúnculo antenal provisto en el segmento basal y en posición ventral, de una fila de seis tubérculos espinosos romos orientados longitudinalmente; segmento intermedio con dos pares de espinas dispuestas en líneas oblicuas relativamente divergentes hacia el margen distal; existen tres espinas en la línea media ventral del tercer segmento distal. En posición dorsal el segmento basal presenta una espina dispuesta longitudinalmente; el segmento medio y el distal poseen dos espinas cada uno, también de orientación longitudinal. Algunas setas plumosas escasas se disponen dorsolateralmente sobre el segundo y tercer segmentos.

Mandíbula (Fig.1c). Con procesos incisor y molar aún no calcificados. La mandíbula derecha posee un diente en el proceso incisor; el borde cortante tiende a recto. El palpo en ambas es desnudo y está formado por tres segmentos cilíndricos subiguales.

Maxílula (Fig.1d). Con endito coxal provisto de 12-14 espinas distales; endito basal con 18-20 espinas relativamente largas en el borde distal y 8-9 en el interno, que se insinúan bajo la cutícula; endopodito con 10-11 setas en el borde interno. Márgenes laterales del basipodito desnudos.

Maxila (Fig.1e). Con enditos digitiformes y largos provistos de 16, 20 y 15 setas muy delicadas dispuestas alrededor de los extremos de éstos y con más de 15 en el borde externo del endopodito. Escafognatito rodeado casi en su totalidad por numerosas setas plumosas.

Primer maxilípedo (Fig.1f). Con endito distal provisto de alrededor de 28 setas pequeñas y marginales insinuándose bajo la cutícula, endopodito pequeño con 8-9 setas plumosas en el margen distal; mitad proximal del exopodito con más de 30 setas plumosas y algunas otras 6 - 7 setas en la base; flagelo del exopodito con 16 segmentos, de los cuales el penúltimo y antepenúltimo son setosos en los márgenes distales. Epipodito lanceolado con algunas setas espaciadas largas, encorvadas y de extremos distales delicadamente aserrados.

Segundo maxilípedo (Fig.1g). Con endopodito provisto de cinco segmentos que presenta 11 espinas en torno a la punta del segmento distal. La mitad proximal del exopodito es de superficie lisa y desnuda; la mitad distal semeja un proceso digitiforme aún no segmentado. El epipodito origina una artrobranquia, algunas setas proximalmente y setas delicadamente aserradas distalmente.

Tercer maxilípedo (Fig.1h). Con endopodito alargado, constituido por cinco segmentos. De éstos el primero posee una línea encorvada ventral provista de 9 tubérculos espinosos, de los cuales los dos distales se ubican en el borde distal interno y constituyen espinas robustas. Desde el segundo al cuarto segmentos están provistos de setas mediales en la mitad distal; quinto segmento con muchas setas en el margen medial. El exopodito posee un segmento basal liso y desnudo, con flagelo digitiforme aún no segmentado. Epipodito similar al del segundo maxilípedo, con una artrobranquia más grande.

Pereiópodos (Fig.1a). Con setas dispuestas espaciadamente en los márgenes ventromediales. El propodito del cuarto y quinto pereiópodos posee una espina roma terminal y ventral; los dactilopoditos son setosos. Del tercero al quinto presentan espinas ventrales pequeñas y delgadas.

Pleópodos (Fig.1i). Tienen basipodito desnudo, endopodito y exopodito lanceolados y setosos, el primero con alrededor de 70 setas y el segundo con 54 , respectivamente. El apéndice interno del endopodito es digitiforme, provisto de 12 pequeños ganchos con forma de anclas (Fig. 1j).

Estadio postpuerulus (Fig.2a). MNHNC D$\mathrm{N}^{\circ} 11099$. Es opaco, de color café chocolate. De forma general muy similar a las langostas adultas. Espinas y setas de tamaño intermedio, totalmente proyectadas fuera del caparazón.

Caparazón subrectangular, notoriamente aplanado. Con rostro corto, robusto y aguzado. Superficie áspera en su mayor extensión, revestido de tubérculos puntiformes muy pequeños. Depresiones de la 
superficie (transformadas en surcos): dos transversales, uno somero ubicado en el límite de las zonas dorsales centrales gástrica y cardíaca y otro posterior, que delimita la elevación que enmarca el límite posterior; un par de surcos longitudinales (relativamente paralelos) delimitan, a cada lado del caparazón, las zonas gástrica y cardíaca de las zonas pleurales laterales.

Región anterior del caparazón con un par de cuernos supraoculares. En vista dorsal se observan como subtriangulares de base ancha y con borde anterior en forma de "S", coronados por una espina aguzada dirigida hacia adelante. Segundo par de espinas bien desarrollado, ubicado inmediatamente detrás de los cuernos, en línea recta con aquéllos. Un par de espinas similares a las señaladas está ubicado lateralmente detrás de cada órbita y otro par se ubica en línea ligeramente oblicua detrás de aquéllas más hacia el centro. Este último par de espinas forma una línea recta con los cuernos supraorbitales y las espinas que se ubican inmediatamente detrás de aquéllos. Las postorbitales forman un triángulo equilátero junto con aquéllas situadas detrás de los cuernos. Los primeros dos pares de espinas submedianas, ubicadas sobre la región gástrica están separadas de las anteriores por una espina central aguzada bien desarrollada y hacia atrás están limitadas por un surco transversal somero; este surco delimita la zona cardíaca en su extremo anterior, la cual está provista de dos filas paralelas de espinas bien desarrolladas, la izquierda con seis y la derecha con siete espinas; las posteriores de ambas filas están ubicadas sobre la elevación posterior. Existen dos espinas anterolaterales, robustas, dirigidas hacia afuera y adelante, las que son muy aguzadas; una espina pequeña se ubica inmediatamente detrás de la segunda. Las espinas anterolaterales están separadas de las grandes espinas dorsolaterales por una constricción del caparazón que es oblicua e inclinada desde el sector lateral hacia el central; estas últimas son muy robustas, cónicas y de base ancha. Detrás de estas últimas se dispone una fila ligeramente encorvada, de 10-11 espinas pequeñas de posición dorsolateral, sobre las regiones pleurales. Elevación transversal posterior con una fila de tubérculos pequeños.

Esternito cefalotorácico subtriangular. Posee una espina frente a la base de las patas ambulatorias primera a cuarta, la espina frente a esta última es robusta; frente a la base de la quinta pata existen dos espinas, la anterior ligeramente más robusta que la posterior. Con cuatro espinas únicas centrales, orien- tadas en la línea media central longitudinal del cefalotórax, desde la primera a la cuarta pata, de tamaño creciente en ese orden. En el borde posterior existen dos espinas centrales pequeñas orientadas hacia atrás.

Abdomen con una carina media dorsal. Primer segmento abdominal con un surco transversal curvo y una expansión triangular lateral y anterior; segundo y tercero con una cimera recta de borde anterior y posterior romos. Cuarto y quinto con una espina muy pequeña en el borde medio distal. Sexto con dos pares de espinas notorias en la superficie dorsal y una espina grande dirigida hacia atrás en el borde posterior y en cada uno de los ángulos laterales posteriores. Áreas pleurales ligeramente elevadas. El primer segmento presenta un borde anterolateral como lóbulo subtriangular y dos lóbulos espinosos de base muy ancha, dirigidos hacia atrás. Bordes laterales segundo al sexto provisto de dos dientes robustos encorvados, dirigidos hacia atrás, de los cuales el anterior es más desarrollado que el posterior. Superficie de los tergitos relativamente áspera, similar a aquélla del caparazón.

Existe un par de espinas sublaterales dirigidas hacia afuera y atrás, presentes sólo en el esternito abdominal de los segmentos primero y sexto.

Telson con cinco espinas dispuestas en la superficie dorsal en línea oblicua dirigida hacia el sector posterolateral.

En este estadio el pedúnculo ocular continúa siendo liso.

Anténulas (Fig. 2b). Cilíndricas y alargadas. Superficie cubierta de setas y espínulas delgadas distribuídas regularmente espaciadas. Pedúnculo constituido por tres segmentos: el segmento distal es igual a $3 / 4$ del basal y el intermedio equivale a $1 / 3$ del segmento basal; el borde distal del tercer segmento se extiende hasta el tercer segmento de la antena y los flagelos sobrepasan ligeramente el borde distal de éste. El segmento basal del pedúnculo antenular es delgado y largo; se extiende, comparativamente, hasta el borde distal del segundo segmento del pedúnculo antenal; provisto de algunas setas suaves, alrededor del estatocisto; posee una línea transversal de casi 9-10 setas, cerca del extremo distal del mismo. Los tres segmentos del pedúnculo están provistos de espínulas cortas y sétulas regularmente espaciadas que dejan superficies relativamente amplias y lisas entre ellas; segmento distal del pedúnculo con una seta simple y larga en la región distal. De los flagelos el externo, que es más 


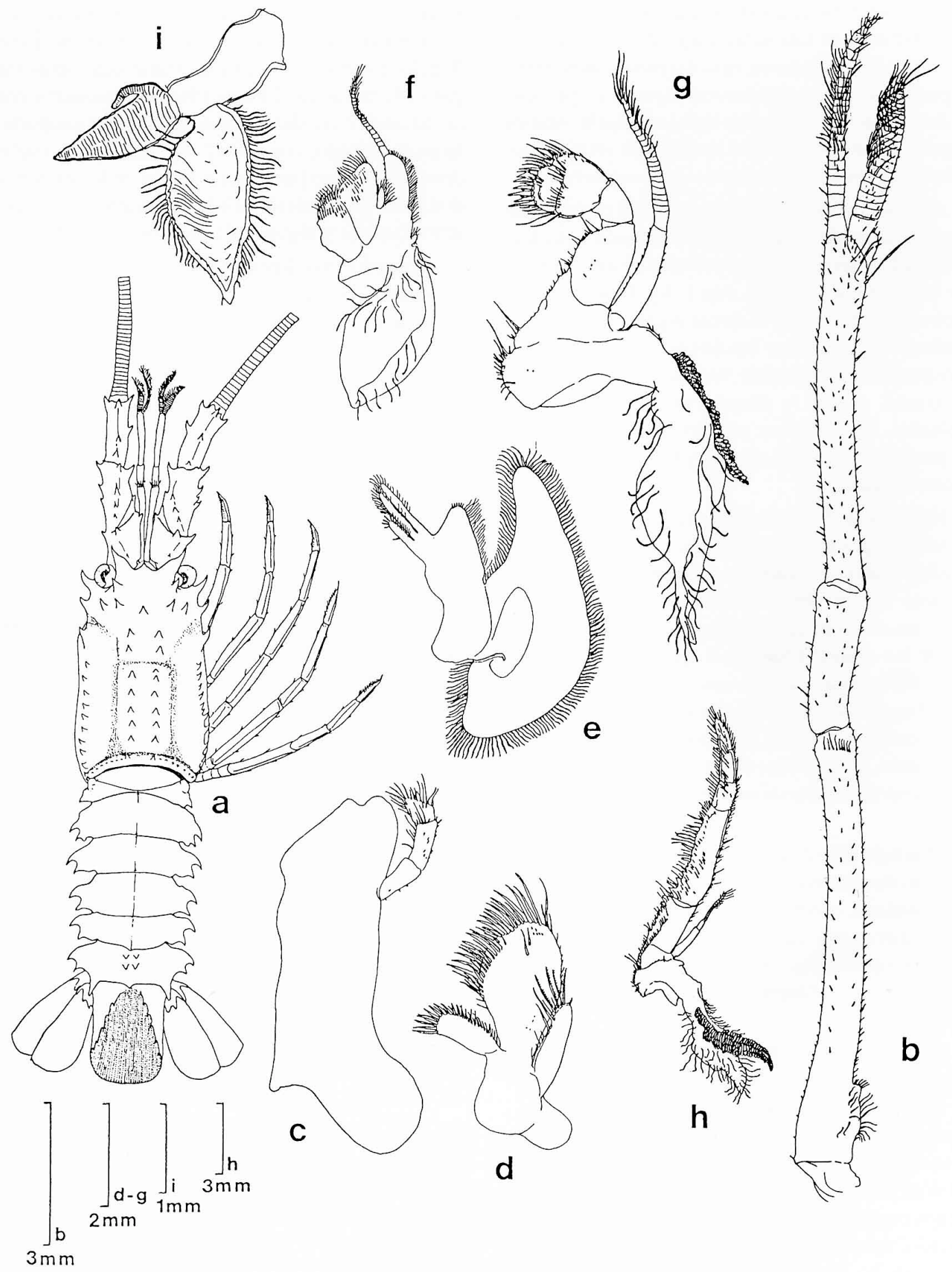

Figura 2. Projasus bahamondei: a, Postpuerulus en vista dorsal; b, anténula; c, mandíbula; d, maxílula; e, maxila; f, primer maxilípedo; g, segundo maxilípedo; $h$, tercer maxilípedo; i, pleópodo.

Figure 2. Projasus bahamondei: a, postpuerulus in dorsal view; b, antennule; c, mandible; d, maxillule; e, maxille; f, first maxilliped; g, second maxilliped; h, third maxilliped; i, pleopod. 
corto y ancho, tiene 23 segmentos, de los cuales el distal termina en tres setas ampliamente divergentes entre ellas; el interno, más delgado y largo tiene un poco más de 26 segmentos, algunos en proceso de división que se observan bajo la cutícula. Ambos flagelos están provistos de abundantes setas sensoriales en los bordes distales de cada segmento.

Antenas. Mayor que la longitud del cuerpo, con flagelos cilíndricos terminados en punta aguzada. Pedúnculo antenal (Fig. 2a) provisto de tres segmentos: basal, medio y distal con 1, 4 y 4 espinas en la línea media dorsal; en el tercer segmento esta línea conforma una carina y las dos espinas medias son muy pequeñas. Superficie ventral de los segmentos proximal, medio y distal con alrededor de 6 espínulas, 3 y 5 espinas, respectivamente. Segmentos provistos con setas escasas y bordes laterales del tercero carinados.

Mandíbula (Fig.2c). Robusta, lisa y larga (cuatro veces equivalente a su ancho): Procesos incisor y molar bien calcificados; con un diente y un borde cortante más o menos recto. Ambas mandíbulas provistas de un exopodito o palpo, de tres segmentos, de los cuales el basal y el segundo son cilíndricos y del mismo tamaño; este último con algunas setas largas hacia el borde interno y distal, y seis setas cortas en el borde externo; el segmento distal, en cambio, es subtrapezoidal, notoriamente más corto, con setas abundantes en los bordes interno y distal.

Maxílula (Fig.2d). Con endito coxal digitiforme, provisto de alrededor de 10 setas espinosas. Endito basal ovalado, con bordes distal e interno continuos y provistos de más de 30 espinas largas; endopodito ovalado con margen interno provisto de numerosas espinas largas. Márgenes laterales del basipodito desnudos.

Maxila (Fig.2e). Con tres enditos digitiformes y notoriamente largos, provistos de 16, 20 y 15 setas muy delicadas y largas, dispuestas en sus contornos del proximal, medio y distal, respectivamente; endopodito triangular con 24 setas largas y delicadas en el borde externo del endopodito y seis pequeñas espínulas apicales. Escafognatito bien desarrollado, más delgado hacia el borde superior, ovalado y ancho hacia el inferior, totalmente rodeado por una cortina continua de setas.

Primer maxilípedo (Fig.2f). Traslúcido. Con endito basal y distal abundantemente setosos, tanto en los bordes como en la porción distal de la superficie interna. Endopodito muy pequeño con 12-13 setas largas; mitad proximal del exopodito con una estructura delicada, relativamente más ancha que el flagelo, totalmente orlada de numerosas setas largas y plumosas en el borde externo proximal y cortas hacia la base del flagelo; flagelo del exopodito largo y delgado, con 21-22 segmentos, los cuales desde el séptimo tienen setas largas en los extremos distales. Epipodito foliáceo, ancho, con una artrobranquia y algunos filamentos finos y largos.

Segundo maxilípedo (Fig.2g). Provisto de un endopodito encorvado, de cinco segmentos; con espinas muy robustas, algunas de las cuales son muy largas en todo el borde exterior del quinto; cuarto segmento provisto en el borde distal, de abundantes espinas largas y setas, mitad proximal desnuda; tercer segmento desnudo; borde interno de los segmentos segundo y basal provistos de setas abundantes en el segundo y aisladas entre las espínulas en el basal. Exopodito cilíndrico, segmentado, más largo que el endopodito, con parte proximal provista de espínulas pequeñas, flagelo con 21-22 segmentos, de los cuales a partir del noveno tienen setas en el borde distal. Segmento basal del apéndice provisto de setas en el borde interno; borde externo liso. Con epipodito provisto de una artrobranquia y setas largas y numerosas.

Tercer maxilípedo (Fig.2h). Con endopodito digitiforme, largo y robusto, formado por cinco segmentos, de los cuales el segundo proximal es el más largo, equivalente a la suma de los tres distales. Todos los segmentos están provistos de setas abundantes, de las cuales hay muchas que son plumosas. En la superficie externa del endopodito hay setas más bien cortas o más cortas que en el borde interno. Borde interno del segmento basal con ocho procesos espinosos y robustos, orientados con sus puntas hacia el interior, de un tamaño creciente, siendo la más reducida la proximal. Segundo segmento con cinco procesos espinosos en el borde interno del tercio proximal, siendo el proceso basal el más desarrollado. El dactilopodito está provisto de espinas abundantes. Exopodito más delgado y corto; se extiende más allá de la base del segundo segmento del endopodito; flagelo con 21-22 segmentos incluidos el basal, que es el más largo, equivalente a la mitad del largo. Posee setas de posición distal en los siete segmentos terminales. Epipodito de un tamaño regular, relativamente más pequeño que el apéndice, provisto de una artrobranquia y con abundantes setas filamentosas.

Pereiópodos. Con estructuras carinadas longitudinales y provistos de setas muy aisladas es- 
pecialmente dispuestas en las superficies ventrales. Los dactilopoditos setosos poseen una espina o garra terminal.

Pleópodo (Fig.2i). Basipodito desnudo, exopodito lanceolado, con escasas setas espaciadas en ambos bordes y en reducción hacia el extremo distal; endopodito digitiforme, sin setas, con apéndice interno encorvado y en reducción.

\section{VARIABILIDAD}

Se observa variabilidad, tanto en los dos ejemplares de estadios puerulus como en los cuatro de postpuerulus estudiados. Ésta se manifiesta principalmente en el desarrollo de las espínulas y espinas de los ejemplares lo que lleva, en ciertos casos, a la impresión errónea de que los diferentes ejemplares de un mismo estadio presentan diferente número de aquéllas. Ejemplifica esta situación el postpuerulus descrito, que presenta nueve espinas en la fila dorsal submediana derecha, en cambio en los otros ejemplares se observan sólo ocho.

Entre las diferencias más notables observadas entre el estadio puerulus y los adultos de $P$. bahamondei están la robustez y desarrollo de las espinas que se extienden sobre el caparazón, tanto las adyacentes a la línea media como aquéllas situadas en los costados pleurales de éste. También es notable la consistencia del exoesqueleto, que con la edad se va tornando más duro y áspero como resultado de la calcificación y aparición de numerosos tubérculos, espínulas, espinas y setas. En el esternito cefalotorácico, existe sólo una espina robusta al frente de las penúltimas patas ambulatorias, a diferencia de los adultos en que existen dos.

Sin embargo, desde un punto de vista relativo son más drásticas y notorias las diferencias que se encuentran entre el puerulus y postpuerulus, que entre este último y los adultos (Tabla 2). En el puerulus las espinas son más romas que en el postpuerulus. Junto a las diferencias señaladas, el postpuerulus se caracteriza porque los tres segmentos que conforman el pedúnculo antenular están cubiertos de espínulas y el primer segmento está provisto de setas en su sector basal en torno al estatocisto, su sector distal posee una fila transversal de nueve setas. Los apéndices ambulatorios se tornan carinados y transversalmente la sección del pedúnculo antenal se torna ovalada, aunque aún no se advierte la fila externo ventral de 8-9, ni la anterolateral de 9 espínulas, característica de los adultos. La superficie dorsal del sexto segmento abdominal presenta los dos pares de espinas mediales que se conservan en el adulto. En el coxopodito del último par de patas ambulatorias se observa una estructura bajo la cutícula, que pudiera corresponder a un gonoporo en formación.

\section{DISCUSIÓN}

Al igual que ocurre con los ejemplares adultos, tanto el puerulus como el postpuerulus de Projasus bahamondei, langosta de Valparaíso o langosta enana, se pueden distinguir fácilmente del puerulus de la langosta de Juan Fernández (Báez, 1979), que es la otra especie de la familia Palinuridae (Jasus frontalis), que vive en aguas de ese archipiélago y en las islas San Félix y San Ambrosio. Los estadios de puerulus y postpuerulus en $P$. bahamondei no han sido reportados del plancton de los alrededores del archipiélago de Juan Fernández (Palma et al., 1976); especie que habita profundidades que se extienden al ámbito de la zona arquibéntica, tanto en las islas como frente a la costa del continente. P. bahamondei y $J$. frontalis pertenecen a los dos únicos géneros que integran el grupo Silentes en esta región del Pacífico. Estos géneros se separaron filogenéticamente en un período geológico muy temprano del resto de los otros palinúridos que pertenecen al grupo de los Stridentes (George y Main, 1967). La superficie del caparazón de $P$. bahamondei se caracteriza por una doble fila de espinas dorsales submedianas y una fila de espinas sobre las regiones laterales branquiales; también presentan una carina abdominal dorsal. Todos estos caracteres son notoriamente diferentes del patrón espinoso que se observa en $J$. frontalis, características que se mantienen desde el puerulus. En los adultos de $P$. bahamondei, tanto el tamaño máximo como el volumen del cuerpo es más reducido que en J. frontalis, aunque a nivel del puerulus y postpuerulus se produce la situación inversa.

Los ejemplares adultos de P. bahamondei se han capturado hasta la fecha como parte de la fauna acompañante en pescas de arrastre de otros crustáceos y a profundidades que oscilan entre 300 y 400 $\mathrm{m}$. También los estadios puerulus y postpuerulus fueron recolectados con artes de arrastre. Esto indicaría que los estadios de esta especie viven en un ambiente fangoso claramente bentónico. Por el contrario, la langosta de Juan Fernández se captura con trampas y el puerulus ha sido capturado en asocia- 
Tabla 2. Projasus bahamondei: Principales diferencias morfológicas observadas entre el puerulus, postpuerulus y adultos.

Table 2. Projasus bahamondei: Main morphological differences observed among the puerulus, postpuerulus and adults.

\begin{tabular}{|c|c|c|c|}
\hline $\begin{array}{c}\text { Estadio } \\
\text { Estructura y característ }\end{array}$ & Puerulus & Postpuerulus & Adultos \\
\hline Consistencia del cuerpo & Flexible y transparente. & Sólida y opaca. & Dura y opaca. \\
\hline Superficie del caparazón & Suave y lisa. & Ligeramente áspera. & $\begin{array}{l}\text { Muy áspera, con espínulas y } \\
\text { tubérculos pequeñísimos. }\end{array}$ \\
\hline Espinas del cuerpo & $\begin{array}{l}\text { Romas, flexibles y frágiles; } \\
\text { no totalmente proyectadas } \\
\text { hacia afuera de la } \\
\text { superficie corporal. }\end{array}$ & $\begin{array}{l}\text { Aguzadas y rígidas; } \\
\text { totalmente proyectadas } \\
\text { hacia afuera de la } \\
\text { superficie corporal. }\end{array}$ & Robustas y gruesas. \\
\hline Caparazón & $\begin{array}{l}\text { Cilíndrico en el dorso; } \\
\text { regiones corporales } \\
\text { delimitadas por depresiones } \\
\text { someras. Espinas } \\
\text { submedianas paralelas. }\end{array}$ & $\begin{array}{l}\text { Aplanado en el dorso; } \\
\text { regiones corporales } \\
\text { delimitadas por surcos muy } \\
\text { anchos. Espinas submedianas } \\
\text { ligeramente divergentes. }\end{array}$ & $\begin{array}{l}\text { Dorso aplanado; regiones } \\
\text { bien delimitadas y } \\
\text { relativamente infladas. } \\
\text { Espinas submedianas } \\
\text { divergentes anteriormente. }\end{array}$ \\
\hline $\begin{array}{l}\text { Primer segmento } \\
\text { abdominal }\end{array}$ & $\begin{array}{l}\text { Con espina dorsal roma, de } \\
\text { base ancha orientada hacia } \\
\text { arriba. Sin surco transversal; } \\
\text { con un tubérculo anterolateral } \\
\text { subtriangular y amplio. }\end{array}$ & $\begin{array}{l}\text { Con tubérculo dorsal somero } \\
\text { y un surco transversal curvo } \\
\text { orientado hacia atrás, que } \\
\text { atraviesa la mitad del } \\
\text { segmento; con un tubérculo } \\
\text { anterolateral globoso } \\
\text { no tan amplio. }\end{array}$ & $\begin{array}{l}\text { Con tubérculo espinoso dorsal } \\
\text { robusto y un surco transversal } \\
\text { curvo y muy amplio orientado } \\
\text { hacia atrás, que atraviesa más } \\
\text { allá de la mitad del segmento; } \\
\text { con un tubérculo anterolateral } \\
\text { triangular. }\end{array}$ \\
\hline $\begin{array}{l}\text { Segundo segmento } \\
\text { abdominal }\end{array}$ & $\begin{array}{l}\text { Con espina dirigida hacia } \\
\text { adelante en borde proximal. }\end{array}$ & Carinado sin espina. & Carinado sin espina. \\
\hline $\begin{array}{l}\text { Cuarto y quinto } \\
\text { segmentos del abdomen }\end{array}$ & $\begin{array}{l}\text { Con espínula media distal } \\
\text { desarrollada. }\end{array}$ & $\begin{array}{l}\text { Con espínula media } \\
\text { distal reducida. }\end{array}$ & Con espina media distal. \\
\hline $\begin{array}{l}\text { Sexto segmento } \\
\text { abdominal }\end{array}$ & $\begin{array}{l}\text { Superficie dorsal con un par } \\
\text { de espinas transversales; una } \\
\text { espina distal central y dos } \\
\text { espinas posterolaterales } \\
\text { a cada lado. }\end{array}$ & $\begin{array}{l}\text { Superficie dorsal con dos } \\
\text { pares de espinas } \\
\text { transversales; una espina } \\
\text { distal central y una } \\
\text { posterolateral a cada lado. }\end{array}$ & $\begin{array}{l}\text { Superficie dorsal con dos } \\
\text { pares de espinas transversales } \\
\text { medias; borde distal con una } \\
\text { espina central y dos } \\
\text { posterolaterales a cada lado. }\end{array}$ \\
\hline Telson & $\begin{array}{l}\text { Superficie lisa, con } 4-5 \\
\text { espínulas en línea oblicua; } \\
\text { superficie sin espínulas. }\end{array}$ & $\begin{array}{l}\text { Superficie áspera, con } 5 \\
\text { espinas en línea oblicua; } \\
\text { superficie sin espínulas. }\end{array}$ & $\begin{array}{l}\text { Con } 5 \text { espinas en línea } \\
\text { oblicua; superficie cubierta } \\
\text { de espínulas. }\end{array}$ \\
\hline Pedúnculo antenal & Sección transversal subcilíndrica. & Sección transversal subovalada. & Sección transversal subovalada. \\
\hline Mandíbulas & $\begin{array}{l}\text { Proceso molar solamente } \\
\text { insinuado; palpo con segmentos } \\
\text { subiguales en forma y tamaño. }\end{array}$ & $\begin{array}{l}\text { Proceso molar pequeño; } \\
\text { palpo con segmentos } \\
\text { diferentes en forma y tamaño. }\end{array}$ & $\begin{array}{l}\text { Proceso molar notorio y } \\
\text { robusto; palpo con segmentos } \\
\text { diferentes en forma y tamaño. }\end{array}$ \\
\hline Maxílulas & $\begin{array}{l}\text { Enditos: distal con } 12-14 \\
\text { espinas; basal con } 18-20 \text { espinas } \\
\text { (borde distal) y } 8-9 \text { (interno). } \\
\text { Endopodito: } 10-11 \text { setas en } \\
\text { borde interno. }\end{array}$ & $\begin{array}{l}\text { Enditos:distal con } 10 \text { espinas; } \\
\text { basal con más de } 30 . \\
\text { Endopodito con más de } 11 \\
\text { espinas en borde interno. }\end{array}$ & $\begin{array}{l}\text { Enditos: distal con algunas } \\
\text { setas largas apicales; basal } \\
\text { totalmente espinoso. } \\
\text { Endopodito sin setas en } \\
\text { borde interno. }\end{array}$ \\
\hline Maxilas & $\begin{array}{l}\text { Endopodito con menos de } 24 \\
\text { setas en borde externo; } \\
\text { sin espínulas apicales. }\end{array}$ & $\begin{array}{l}\text { Endopodito con } 24 \text { setas en } \\
\text { borde externo; con seis } \\
\text { espínulas apicales. }\end{array}$ & $\begin{array}{l}\text { Endopodito setoso en } \\
\text { borde externo y región apical. }\end{array}$ \\
\hline Pleópodos & $\begin{array}{l}\text { Exopodito y endopodito } \\
\text { ovalados, alargados y subiguales. } \\
\text { Con ganchitos en borde distal } \\
\text { de apéndice interno no modificado. }\end{array}$ & $\begin{array}{l}\text { Exopodito más largo y más } \\
\text { ancho que el endopodito. Sin } \\
\text { ganchitos en borde distal de } \\
\text { apéndice interno modificado. }\end{array}$ & $\begin{array}{l}\text { Exopodito y endopodito } \\
\text { foliáceos, de forma y } \\
\text { estructura diferentes según } \\
\text { sexo. }\end{array}$ \\
\hline
\end{tabular}


ción con ese arte de pesca. Por esta razón, sería posible que el número de ejemplares capturados pudiera aumentar si en su captura se utilizaran artes estáticos más apropiados para la captura de palinúridos (Báez y Ruiz, 1985).

Los estadios puerulus fueron recolectados en primavera y los postpuerulus en invierno, lo que indicaría que durante el semestre de verano-otoño ocurrió solamente una muda.

El puerulus de $P$. bahamondei, aunque manifiesta características claramente larvarias como es el apéndice interno del endopodito de los pleópodos, característica que le permitiría nadar incorporado en el ambiente planctónico, difiere notoriamente de las larvas filosomas de hábitos claramente planctónicos. Por el contrario, presenta una notable semejanza en su morfología general con los ejemplares adultos. Báez (1973) describió una larva filosoma, "Phyllosoma X", la que fue asignada tentativamente a P. parkeri. Sin embargo, Webber y Booth (1988) sugirieron que el citado último estadio "phyllosoma X" no correspondería al de P. bahamondei. Aunque la posibilidad de una determinación errónea de la filosoma aludida no puede ser descartada del todo, ninguna otra especie de palinúrido ha sido reportada, tanto de aguas en torno a los archipiélagos como de la zona arquibéntica, que se extiende frente a Chile continental. En consecuencia, sería posible afirmar que los estadios puerulus y los drásticos cambios morfológicos que se observan derivarían de la última muda de esa "Phyllosoma X" en su metamorfosis al puerulus de $P$. bahamondei.

Existen claras diferencias morfológicas entre el puerulus descrito por Webber y Booth (1988) asignado a $P$. parkeri y los puerulus de $P$. bahamonde $i$ aquí descritos. Estas diferencias se manifiestan principalmente en la disposición de las filas de espinas de las regiones branquiales del caparazón, las que son más encorvadas en esta última. También es notoria la presencia de dos pares de espinas en la superficie dorsal del sexto segmento abdominal de $P$. parkeri y sólo un par en $P$. bahamondei. En la figura del puerulus de $P$. parkeri se observa el pedúnculo antenular formado por cuatro segmentos; $P$. bahamondei, en cambio, tiene sólo tres segmentos.

\section{AGRADECIMIENTOS}

Al Dr. Sergio Zunino, Investigador del Museo de Historia Natural de Valparaíso, Sr. Alejandro González, por las muestras legadas y al Sr. Patricio
Zavala F., Museólogo de la Pontificia Universidad Católica de Chile, por la valiosa colaboración prestada a la realización de este trabajo.

\section{REFERENCIAS}

Báez, P. 1973. Larvas phyllosoma del Pacífico Sur Oriental (Crustacea, Macrura, Scyllaridea). Rev. Biol. Mar., 15(1): 115-130.

Báez, P. 1979. El puerulus de Jasus frontalis (H. Milne Edwards, 1837) (Crustacea: Decapoda: Palinuridae). Rev. Biol. Mar., 16(3): 225-228.

Báez, P. y R. Ruiz. 1985. Crustáceos de las islas oceánicas de Chile depositados en el Museo Nacional de Historia Natural de Santiago. En: P. Arana (ed.). Investigaciones Marinas en Archipiélago de Juan Fernández. Esc. Ciencias del Mar, UCV, Valparaíso, pp. 93-108.

George, R.W. 1976. A new species of spiny lobster, Projasus bahamondei (Palinuridae "Silentes"), from the south east Pacific region. Crustaceana, 30(1): 27-32.

George, R.W. y A.R. Main. 1967. The evolution of spiny lobsters (Palinuridae): a study of evolution in the marine environment. Evolution, 21: 803-820.

Palma, S., J. Meruane y A. Mujica. 1976. Observaciones sobre el meroplancton del archipiélago de Juan Fernández. Cienc. Tec. Mar., CONA 2: 117 126.

Retamal, M.A. y P. Arana. 2000. Descripción y distribución de cinco crustáceos decápodos recolectados en aguas profundas en torno a las islas Robinson Crusoe y Santa Clara (Archipiélago de Juan Fernández, Chile). Invest. Mar., Valparaíso, 28: $149-163$.

Webber, W.R. y J.D. Booth. 1988. Projasus parkeri (Stebbing, 1902) (Crustacea, Decapoda, Palinuridae) in New Zealand and description of a Projasus puerulus from Australia. Nat. Mus. N.Z. Records, 3(8): 81-92.

Weinborn, J.A., P. Báez y A. Radtchenko. 1992. Pesca oceánica: Langosta en el Mar Presencial. Rev. Chile Pesquero, 67: 21-24. 